\title{
Electric Field Induced Magnetic Switching At Room Temperature: Switching Speed, Device Scaling and Switching Energy
}

Khalid Ashraf*, Samuel Smith and Sayeef Salahuddin

Department of Electrical Engineering and Computer Sciences, University of California, Berkeley, CA 94720 USA

"E-mail: kashraf@eecs.berkeley.edu

\section{Abstract}

The switching energy, speed and scaling behavior are calculated for a magneto-electric device incorporating the multiferroic material bismuth ferrite (BFO). For this purpose, a massively parallel phase field model is developed for simulation of the device. First, multidomain switching of thin film ferroelectric is shown to match with experimental measurements. We show that the switching energy in these devices can be an order of magnitude lower than other alternative approaches for magnetization reversal. We also show that the coercive voltage scales almost linearly in scaled BFO islands; however, the switching speed requires further study.

\section{Introduction}

A magnetoelectric device is desired for electric field control of magnetism. There are a number of proposed methods for achieving such functionality that are under investigation. A material level realization of magneto-electric coupling can be achieved in the multiferroic material $\mathrm{BiFeO}_{3}$. However, $\mathrm{BFO}$ is an antiferromagnet and hence the reversal of the polarization does not facilitate a magnetic moment reversal. An alternative route is to couple a ferromagnet with the antiferromagnet using exchange bias. Recently, the complete reversal of a magnetic moment with the application of an electric field has been demonstrated in a ferromagnet (FM)- $-\mathrm{BiFeO}_{3}$ heterostructure [1]. However, for practical device applications, there still remains many unknowns about the physical mechanism of the device operation and the associated energetics. We estimated the magnetic energy values of the $\mathrm{BiFeO}_{3}-\mathrm{CoFeB}$ heterostructure and found the quantum mechanical exchange energy to be strong enough to induce ferromagnetic reversal [2]. We also developed a massively parallel phase field model to study the correlated switching dynamics of thin film multidomain ferroelectrics like $\mathrm{BiFeO}_{3}$ and explained a number of intriguing domain switching processes that were not understood previously [1,3]. In this work, we analyze the energy dissipation and switching speed of a magnetoelectric device using our experimentally benchmarked model. We show that (i) incorporation of inhomogeneous strain in a multi-domain switching process predicts the correct experimental coercive field of thin film $\mathrm{BiFeO}_{3}$ and (ii) an inhomogeneous electric field distribution due to an AFM tip qualitatively describes the experimental domain wall velocity for a range of applied fields. (iii) We show that in scaled nanoscale islands, the coercive voltage scales almost linearly and (iv) finally, for the first time, that the estimated per unit area energy dissipation in these devices can be up to one order of magnitude lower compared to the alternative magnetic switching routes like the spin transfer torque devices.

\section{Device Structure and Theory}

A typical magnetoelectric device is shown in Fig. 1. A magnetic nanodot is placed on top of the BFO thin film. Electric field is applied on the magnetic dot that switches the polarization in BFO that in turn switches the magnet. The polarization directions in a BFO unit cell are shown in Fig.2. Three different surfaces of BFO are studied in this work. They are (001), (110), and (111). The magnetic energy scale is orders of magnitude lower than the ferroelectric energy scale. The switching energy and dynamics are practically determined by the polarization dynamics in these systems. Hence, in this work, we will only focus on the switching dynamics and energy dissipation in the ferroelectric material.

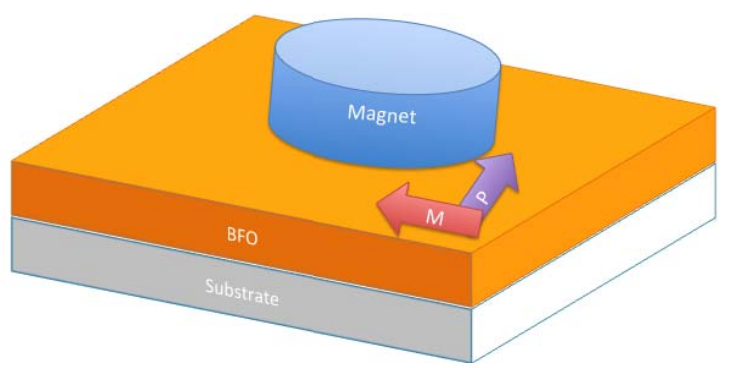

Fig 1. A schematic of the device structure. A thin film of BFO is grown on a substrate. A CoFeB magnet is patterned on $\mathrm{BFO}$ and used as a top electrode. When a voltage pulse is applied on the electrode, the polarization in the multi-ferroic material switches, and with it, the magnetic moment in the ferromagnet switches.

The BFO unit cell is rhombohedral with eight possible polarization orientations. When a vertical electric field is applied, the polarization switches by the reversal of one, two, and three components of the polarization on the (001), (110), and (111) surfaces respectively.
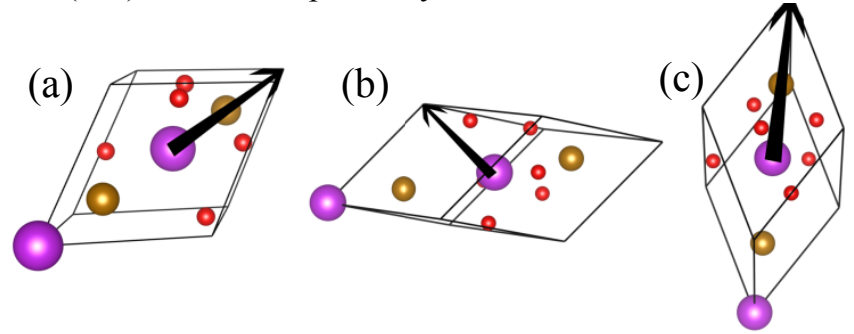

Fig 2. The relative orientations of the polarization on the (a) (001), (b) (110), and (c) (111) surfaces of BFO.

The ferroelectric device is studied using a phase field model. A phase field model describes the thermodynamic free energy of a system in terms of a continuous field variable. For our ferroelectric system, the contributing factors to the system 
energy were chemical energy, electrostatic energy, elastic energy, domain wall energy and thermal energy. Once all of these energies are taken into account, the temporal evolution of the system is then calculated with the time-dependent Ginzberg-Landau equation. The details can be found in ref. [3].

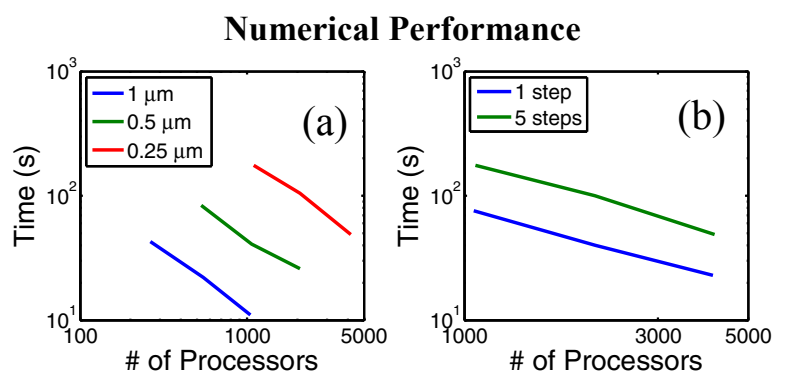

Fig. 3. Phase field code scaling. Micron scale devices with $\mathrm{nm}$ grid resolution are simulated. (a) The code shows linear scaling up to 5000 processors. (b) Further improvement in performance can be achieved by calculating the long-range interactions every 5 steps instead of every single step. This assumption does not change the results obtained from the calculation.

The code was implemented using the numerical solver PETSc. Mixed finite difference and finite element (FEM) grids were used for the simulation of electrostatics and strain calculation respectively. A novel node wise assembly algorithm was applied for assembling the structure stiffness matrix [3]. A novel method of coupling phase field model with FEM was developed. An isoparametric 3D linear brick element was used as the element in the FEM calculation. The nonlinear strain at the nanoscale is calculated using the eigen strain method. A velocity verlet integration algorithm was used for time marching the PDE solutions. The code performance scales linearly over thousands of CPUs and is capable of capturing the physics of micron scale devices that are fabricated experimentally.

\section{Results: Multi-domain Hysteresis}
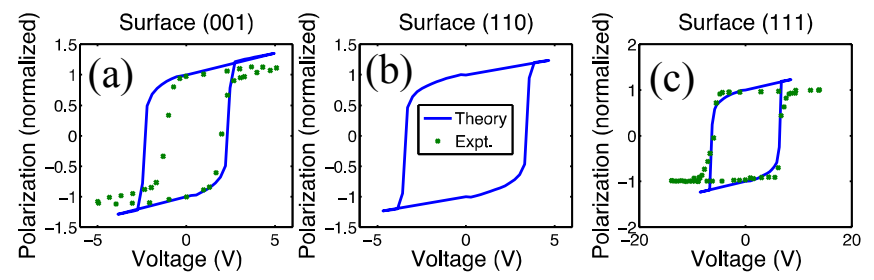

Fig 4. Coercive Field: Multi-domain phase field simulation of the polarization vs. voltage loop of BFO on (a) (001), (b) (110), and (c) (111) surfaces. Experimental data on the (001) and (111) surfaces are shown for comparison. Multi-domain calculation shows excellent agreement with the experimental coercive field.
A monodomain calculation of the BFO thin film overestimates the coercive field by an order of magnitude. This is because in real ferroelectrics, switching is thermally activated at the domain wall boundary. Inclusion of domain walls in the film reduces the coercive field at the wall. When an electric field is applied, switching begins at the nucleation center located randomly on a domain wall. The domain then grows under the applied field. A multi-domain calculation including strain and electrostatic energy shows excellent agreement with the shape and coercive field of the hysteresis loop. Agreement between the calculated and experimental coercive fields indicates that the BFO thin film is multidomain. The almost square hysteresis loops of BFO in a multidomain configuration is an indication that the domain switching speed in BFO is significantly high compared to the voltage ramp rate. Also the coercive field increases gradually from (001) to (110) to the (111) surface.

\section{Results: AFM induced switching}
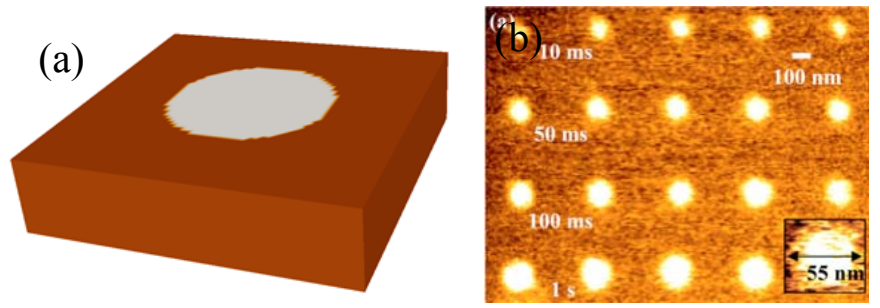

Fig. 5. Switching of the ferroelectric domains in (a) simulation and (b) experiment. The voltage is applied using an AFM tip.

Domain growth velocity under an electric field is difficult to characterize since in a multi-domain film, the initial distribution of the domains and their coupled dynamics is a complex process. A popular method of estimating the domain wall velocity is to apply a local voltage using an AFM tip. The radius of the single switched domains after varying length of the voltage pulse can be measured to obtain an estimation of the domain wall velocity under the applied voltage. A simulation of an AFM switched domain is shown with the PFM image of an experimentally switched domain. The circular growth of the simulated domain is due to the hemispherical potential profile 'M tip that is used to switch the domain. sal vertical domain is nucleated under the is all the way to the bottom electrode. domain grows radially outward. The mainly determined by the speed of this radial growth of the domain.

\section{Results: Domain velocity and activation field}

Experimentally observed domain evolution shows a highly nonlinear behavior as shown in Fig. 6. We calculated the domain diameter as a function of time at different applied voltages. On the (001), (110) and (111) surfaces, the 

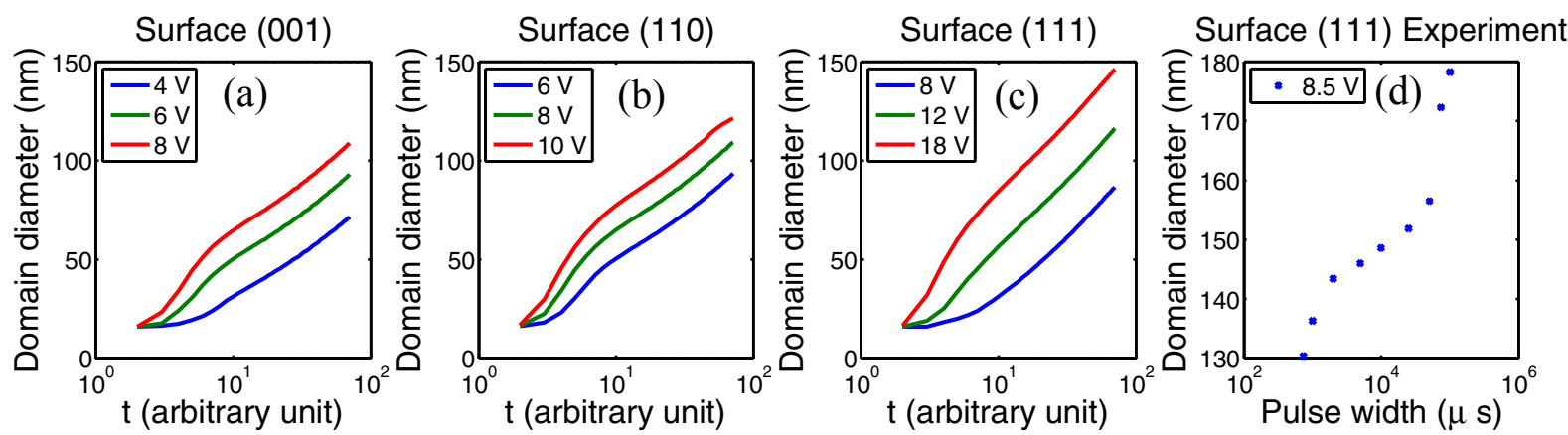

Fig 6. Domain Wall Velocity: The simulated domain wall velocity as a function of electric field is calculated on the (a) (001), (b) (110), and (c) (111) surfaces of BFO. (d) The experimental domain diameter as a function of the pulse width is also shown. The voltage was applied using an AFM tip. As expected, the domain wall velocity increases with increasing applied field on all the surfaces. The functional behavior of the experimental curve on the (111) surface shows resemblance to the high voltage growth in (c).

polarizaion has to switch one, two and three components respectively. The speed of the domain wall increases from (001) to (110) to (111). The switching speed is calculated in arbitrary time scale. The phase field model describes the domain dynamics at a diffusive limit. The exact switching speed depends on the dimensionality, material parameters and boundary conditions. Thus it is not possible to calculate the switching speed in an ab-initio method. However, the speed can be extracted from the experimental data. The experimental data shown in Fig. 6 is in the millisecond regime. Here, the switching is performed at an adiabatic limit. The intrinsic switching in ferroelectrics can be significantly faster. Picosecond switching has been reported in other ferroelectric materials [6]. The polarization dynamics and magneto-electric coupling at this time scale remain to be understood.
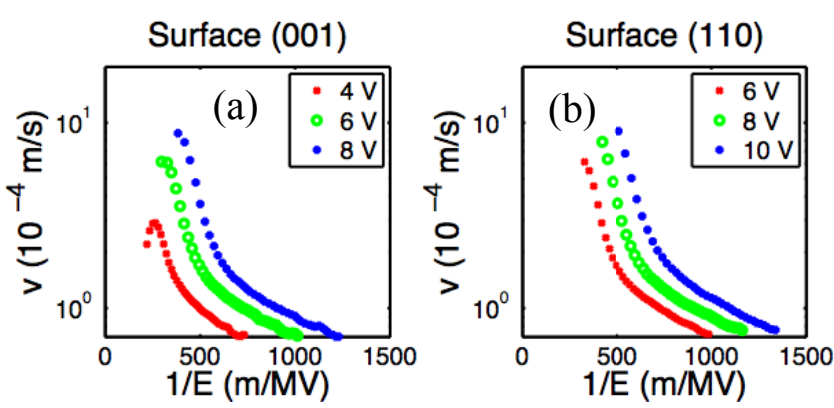

qualitative agreement with the simulated domain growth. The domain wall velocity increases exponentially with the increase of the applied field. Also at the same applied field, the domain wall velocity is higher for higher applied voltage. This result is not directly evident from Merz's law. This trend is seen in the experimental data as well. Our multi-scale model is able to capture this effect since the inhomogeneous electric field is included in the calculation. With increasing applied voltage, the lateral component of the electric field increases and thus the domain wall velocity is higher for higher applied voltages.

Fig 7. Activation Barrier: The domain wall velocity as a function of electric field is calculated for the (a) (001), (b) (110), and (c) (111) surfaces of BFO. (d) The experimental domain wall velocity versus the applied electric field is also shown for comparison. Although, the experimental velocity changes by almost five orders of magnitude, the general trend of the velocity scaling with applied field is captured in the simulation results.

Domain wall switching in multi-domain ferroelectrics is an activated process that is qualitatively described by Merz's law. Here, we have used an atomistic description of the domain wall velocity as shown in Fig. 7. For these plots, the electric field for each time step was calculated as the vertical field from the AFM tip at the moving domain wall boundary position. The activation energy for domain propagation is a maximum on the (111) surface shown in Fig. 7(c). Experimental data on the (111) surface from Ref. [5] is also shown for comparison and shows
We calculated the coercive voltage of a $40 \mathrm{nmX} 40 \mathrm{~nm}$ island with varying thickness. We find that the voltage scaling shows almost linear trend as a function of island thickness. The coercive voltage of (111) surface is higher than the (001) surface for all the thickness ranges. The switching field for the mono-domain sample is higher than any multi-domain sample. Note that the slope of the voltage versus thickness curve 

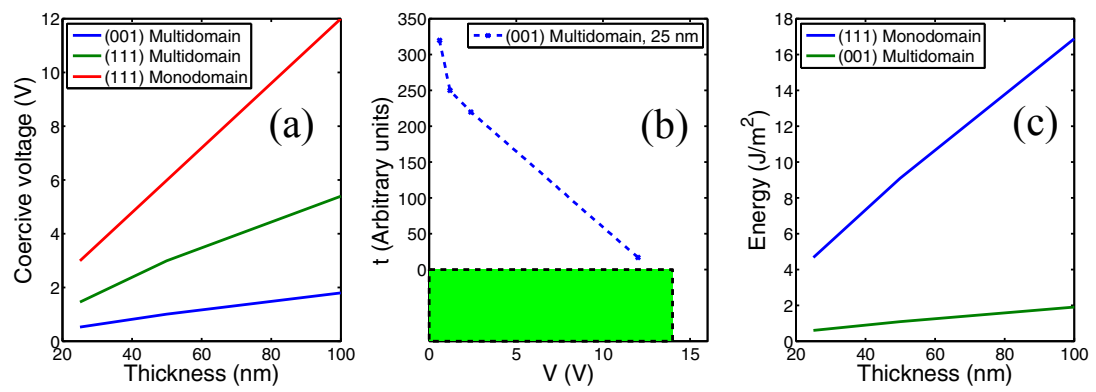

Fig 8. Coercive voltage scaling and energy dissipation in a nanoisland: (a) The scaling of coercive voltage as a function of film thickness. Coercive voltage shows almost linear scaling with film thickness. (b) Decrease of switching with increasing applied voltage. Within the range of simulation time, the voltage increases by more than 10 times for speed increase of 20 times. (c) Switching energy versus thickness for (001) multidomain and (111) monodomain BFO. Only $\sim 0.6 \mathrm{~J} / \mathrm{m}^{2}$ is dissipated for adiabatic switching on (001) surface $25 \mathrm{~nm}$ thick device. The switching energy increases to about $5 \mathrm{~J} / \mathrm{m}^{2}$ for fast switching in (111) monodomain sample at $25 \mathrm{~nm}$ thickness.

increases with increasing coercive voltage. The slight nonlinearity of the voltage scaling arises from the inhomogeneous electric field present in the device during switching. The almost linear scaling of coercive voltage with thickness is an indication that at the nanoscale, BFO retains its polarization. This is due to the significantly lower strain to polarization coupling in $\mathrm{BFO}$ compared to other ferroelectrics like $\mathrm{PbZr}_{\mathrm{x}} \mathrm{Ti}_{1-\mathrm{x}} \mathrm{O}_{3}$.

The coercive voltage shown in Fig. 8(a) is at the adiabatic limit. Fig. 8(b) shows the switching time versus the applied voltage for the multi-domain (001) BFO at 25nm thickness. For a voltage change of $\sim 10$ times from $\sim 1 \mathrm{~V}$ to $\sim 12 \mathrm{~V}$, the switching time decreases by $\sim 30$ times from $\sim 320$ to $\sim 10$ in arbitrary units. Although this is more than an order of magnitude reduction in switching time, still comparison to experiment would predict a switching time in the microsecond regime. Notably, picosecond switching has been demonstrated in ferroelectric materials [6]. Our calculations show that dynamics at these short time scales cannot be fully domain wall mediated and it is a question that remains to be explored.

Finally, we show the switching energy versus thickness for the 40nmX40nm island as shown in Fig 8(c). At the adiabatic limit, the switching energy is $\sim 0.6 \mathrm{~J} / \mathrm{m}^{2}$ for the (001) multi-domain device at $25 \mathrm{~nm}$ thickness. When a monodomain (111) device is used, the switching energy increases to $\sim 5 \mathrm{~J} / \mathrm{m}^{2}$. This is to be compared with the most optimized result in spin transfer torque device where magnetic switching with areal energy density of $30 \mathrm{~J} / \mathrm{m}^{2}$ has been demonstrated [7]. Note that the two curves in Fig. 8(c) provide two limiting cases in terms of switching speed. For the green curve, the switching energy is very small but the speed is also very slow. On the other hand, for the blue plot, the switching could be very fast $(<100$ ps [6]), but it comes at the cost of increased energy. Nonetheless, even at this increased energy, high speed limit, the dissipation is almost an order of magnitude smaller than a highly optimized STT device [7]. Further optimization of energy-delay metric could be possible by appropriately doping $\mathrm{BFO}$ that reduces its coercive field while retaining the magnetic moment.

\section{Conclusion}

In conclusion, we have shown that a phase field calculation of multidomain switching process predicts the correct experimental coercive field of thin film $\mathrm{BiFeO}_{3}$. Also a lateral electric field distribution due to an AFM tip qualitatively describes the experimental domain wall velocity for a range of applied fields. We show that in scaled nanoscale islands, the coercive voltage is reduced significantly and the polarization in the film is retained due to the low strain-polarization coupling in BFO. Finally, for the first time, that the estimated per unit area energy dissipation in these devices can be more than one order of magnitude lower compared to the alternative magnetic switching routes like the spin transfer torque devices at the adiabatic limit. Further understanding of switching at high fields is necessary and is currently under investigation.

\section{Acknowledgement}

Fruitful discussions with J. Heron, M. Trassin and R. Ramesh are acknowledged. This work was supported in part by Nanoelectronic Research Initiative (NRI) and National Science Foundation (NSF). The computer simulations were performed at the National Energy Research Scientific Computing Center (NERSC).

\section{References}

[1] J. Heron, et al., PRL, 89, 153504 (2011).

[2] K Ashraf, S. Salahuddin JAP, 111(10), 103904 (2012).

[3] K Ashraf, S. Salahuddin JAP, (accepted) (2012).

[4] J. Wang et al., Science, v 299, 1718, (2003).

[5] Y. C. Chen et al., APL, 94, 122908 (2009).

[6] J.Li et al. APL,84,7, 1174, (2004).

[7] S. Ikeda et al., Nat. Mater. 9, 721-724 (2010). 